\title{
Fracturas en niños: experiencia en un centro de alta complejidad del suroccidente Colombiano
}

\author{
Fractures in children: experience in a tertiary \\ referral hospital in South west Colombia
}

\author{
Juan-Pablo Martínez-Cano ${ }^{1,2}$; Laura Zamudio-Castilla ${ }^{1}$; Julio-César Mantilla²; Diana-Carolina Caicedo²; \\ Daniel Vernaza-Obando ${ }^{2}$; Alfredo Martínez-Rondanelli ${ }^{1,2}$
}

Forma de citar: Martínez-Cano JP, Zamudio-Castilla L, Mantilla JC, Caicedo DC, Vernaza-Obando D, Martínez-Rondanelli A. Fracturas en niños: experiencia en un centro de alta complejidad del suroccidente Colombiano. Rev Univ Ind Santander Salud. 2019; 50(4): 309-315. doi: http://dx.doi.org/10.18273/revsal.v51n4-2019004 (c) (1)

\section{Resumen}

Introducción: Es importante conocer las características demográficas y clínicas de las fracturas en niños para mejorar las estrategias de prevención y tratamiento en estos pacientes. Objetivos: Describir las fracturas en niños y sus características epidemiológicas. Metodología: Estudio tipo corte transversal desde enero 2014 hasta diciembre 2017. Pacientes menores de 18 años que consultaron por fracturas a una clínica en Colombia fueron incluidos. Se incluyeron datos demográficos, lugar donde ocurrió el trauma, huesos fracturados y tipo de tratamiento. Se evaluó la distribución por género y grupo etario. La fuente de información fue la historia clínica. Se utilizaron medidas de tendencia central y dispersión para agrupar los datos. Se calculó la incidencia de fracturas por cada 1000 niños/ año. Este estudio recibió aval del comité de ética de la institución. Resultados: Hubo 2436 niños fracturados, $65.6 \%(\mathrm{n}=1597)$ ocurrieron en hombres. La mayoría fueron entre 6-11 años $(40.7 \%, \mathrm{n}=991)$, luego entre $12-17$ años $(36.4 \%, \mathrm{n}=887)$ y finalmente, menores de 6 años $(22.9 \%, \mathrm{n}=558)$. La localización de la fractura más frecuente entre $0-5$ años fue el húmero $(30.6 \%, \mathrm{n}=171)$, seguido por el radio $(29 \%, \mathrm{n}=162)$ y la clavícula $(15.9 \%, \mathrm{n}=89)$; entre 6-11 años fue el radio $(45.2 \%, \mathrm{n}=448)$, seguido por el húmero $(18 \%, \mathrm{n}=178)$ y el cúbito $(16.6 \%, \mathrm{n}=165)$; y entre $12-17$ años fue el radio $(34.6 \%, \mathrm{n}=307)$, seguido por los huesos de la mano $(22.7 \%, \mathrm{n}=201)$ y los huesos del pie $(10.8 \%, n=96)$. Requirieron manejo quirúrgico $30.9 \%$ de los niños fracturados $(n=752)$. La incidencia de fracturas fue de 29.7/1000 niños por año. Conclusión: Las fracturas en niños se presentan con mayor frecuencia en el género masculino. Los huesos fracturados varían dependiendo del grupo etario, con un porcentaje importante del radio en todos los grupos. La mayoría se manejan de forma conservadora.

Palabras clave: Fracturas óseas; Niño; Epidemiología; Reducción cerrada; Reducción abierta; Fijación de fractura.

1. Fundación Valle del Lili. Cali, Colombia.

2. Universidad Icesi. Cali, Colombia.

Correspondencia: Juan Pablo Martínez Cano. Dirección: Fundación Valle del Lili, Departamento de Ortopedia, Cra 98 No. 18-49 Of. 118, Cali. Teléfono: +5723319090. Correo electrónico: jpmartinezc@gmail.com 


\begin{abstract}
Introduction: It is important to know the demographic and clinical characteristics of fractures in children to improve prevention and treatment strategies in these patients. Objectives: To describe fractures in children and their epidemiological characteristics. Methodology: Cross-sectional study performed between January 2014 and December 2017. Patients under 18 years old with fractures who consulted to a hospital in Colombia were included. Demographic data, the location where the trauma occurred, fractured bones and type of treatment were included. Age and gender distribution were analyzed. Medical records were the source of information. Central tendency and dispersion measures were used to group the data. Incidence of fractures per 1000 children / year was calculated. This study received approval from the institution's ethics committee. Results: There were 2436 children with fractures, $65.6 \%(\mathrm{n}=1597)$ occurred in males. Most fractures were in children between $6-11$ years old $(40.7 \%$, $\mathrm{n}=991)$, followed by $12-17$ years old $(36.4 \%, \mathrm{n}=887)$ and finally by children under 6 years old $(22.9 \%, \mathrm{n}=558)$. For the group under 6 years, most fractures occurred in humerus $(30.6 \%, \mathrm{n}=171)$, followed by radius $(29 \%, \mathrm{n}=162)$ and clavicle $(15.9 \%, n=89)$; between $6-11$ years old it was in radius $(45.2 \%, n=448)$, followed by humerus $(18 \%$, $\mathrm{n}=178)$ and ulna $(16.6 \%, \mathrm{n}=165)$; and between $12-17$ years it was in radio $(34.6 \%, \mathrm{n}=307)$, followed by bones of the hand $(22.7 \%, n=201)$ and bones of the foot $(10.8 \%, n=96)$. Surgical treatment was required in $30.9 \%(n=752)$ of the patients. The incidence of fractures was 29.7/1000 children per year. Conclusions: Fractures in children occur more frequently in males. The fractured bones differ depending on the age group; however, radius fractures represent an important proportion in all groups. Most fractures in children are treated in a conservative manner.
\end{abstract}

Keywords: Fractures; Bone; Infant; Child; Preschool; Adolescent; Closed fracture reduction; Open fracture reduction; Fracture fixation.

\section{Introducción}

En los países en vía de desarrollo, la principal causa de morbimortalidad en la población menor de 18 años son las lesiones traumáticas ${ }^{1-3}$. De estas, las fracturas constituyen la causa más frecuente, siendo un tercio de todas las consultas por trauma en niños ${ }^{4,5}$. La incidencia mundial de fracturas es de alrededor de 20 por cada 1000 niños $^{6-8}$. Se considera que $25-30 \%$ de los niños presentarán al menos una fractura antes de la vida adulta ${ }^{9,10}$, convirtiéndose así, en un problema de salud pública.

En diferentes continentes se ha encontrado que la mayoría de las fracturas en niños se presentan en el hogar ${ }^{7,11,12}$, siendo únicamente el $25 \%$ presenciadas por los padres ${ }^{13}$. Le siguen los accidentes escolares (13\%) y los accidentes de tránsito $(12 \%)^{14}$. Además, son más comunes en niños (66\%) que en niñas $(34 \%)^{8,14-16}$.

Cerca de $66 \%$ de las fracturas pediátricas se localizan en los miembros superiores ${ }^{9}$, mientras el $21 \%$ ocurren en los miembros inferiores ${ }^{17}$. En la literatura se ha descrito que el sitio anatómico más común es el antebrazo (37\%), siendo el radio distal la fractura más común de la población pediátrica ${ }^{4,18,19}$.

Para 1990, la incidencia anual calculada de fracturas en niños en Cali era de 6.17 por cada 1000 niños ${ }^{20}$.
Este estudio incluyó únicamente menores de 14 años, excluyendo a los adolescentes. Durante la infancia, las caídas de propia altura corresponden a la causa más común de fractura (54\%), pero a los 17 años, solo causan el $6 \%$ de las mismas, siendo más frecuente el trauma deportivo y los accidentes de tránsito ${ }^{15}$.

En general, las fracturas en niños se manejan ortopédicamente. Solo $10 \%$ requieren tratamiento quirúrgico ${ }^{14}$. La necesidad de admisión por fractura en menores de 14 años varía de $38-71 \%{ }^{17,21}$. Otros estudios reportan tasas más bajas, con $86.6 \%$ manejadas ambulatoriamente ${ }^{21-25}$.

Describir la epidemiología de las fracturas infantiles aporta conocimiento respecto a los mecanismos, la etiología, los huesos comprometidos, el tipo de tratamiento y los lugares donde ocurren estos traumas. Esta información puede ayudar a introducir estrategias preventivas que disminuyan el número de niños lesionados.

\section{Objetivos}

Realizar una descripción epidemiológica de las fracturas en niños atendidas en un centro de alto nivel de complejidad en Colombia y evaluar la asociación con variables como género y rango de edad. 


\section{Materiales y Métodos}

Estudio tipo corte transversal que incluye todos los pacientes menores de 18 años que consultaron por urgencias o consulta externa, a un centro médico de alta complejidad en Colombia presentando una o más fracturas. El estudio se desarrolló entre enero 1 de 2014 y diciembre 31 de 2017. Se excluyeron únicamente los pacientes con fracturas en cara o cráneo.

\section{Extracción de la información}

A través del departamento de estadística de la institución se realizó una búsqueda de los pacientes con códigos de la Clasificación Internacional de Enfermedades $10^{\mathrm{a}}$ versión (CIE-10) que correspondían a fracturas óseas de todo el cuerpo, exceptuando cara y cráneo. En total se utilizaron 73 códigos diferentes. Las historias clínicas electrónicas fueron evaluadas en el sistema SAP. Los datos de interés fueron tomados y registrados en la base de datos previamente diseñada en el sistema BD Clinic.

\section{Análisis de los datos}

En el análisis, se utilizaron medidas de tendencia central y dispersión para agrupar los datos cuantitativos. La distribución de las variables se calculó con la prueba de Shapiro-Wilk, encontrándose que no cumplían criterios de normalidad, por lo que se les aplicó mediana y rango intercuartílico. Las variables cualitativas se presentan en porcentajes. Se calculó la incidencia por cada 1000 niños, a partir del total de niños fracturados cada año, sobre el total de niños que consultaron a la institución en el mismo año. Esto como una aproximación a la incidencia poblacional, teniendo en cuenta que la zona de influencia de la clínica es muy similar al total de niños que consultan en los servicios de urgencias y consulta externa de la misma. Para el análisis de los datos se utilizó el Software STATA 12.1.

\section{Resultados}

Durante los cuatro años del estudio, ingresaron 2436 niños con 2821 fracturas, para un promedio 705 fracturas cada año. El $65.6 \%$ fueron varones. La mediana para la edad fue 10 años, con un pico de mayor incidencia de fracturas entre los 12 y 13 años. La mayoría de los pacientes (78.8\%) eran procedentes de la ciudad de Cali, el 14\% vivía en otros municipios del Valle y el 7.2\% restante vivía en otros departamentos. Las características demográficas se pueden ver en la Tabla 1.
El colegio fue el sitio donde más se fracturaron los niños (20.6\%), seguido por la escuela de fútbol $(17.7 \%)$ y el hogar (16\%). Respecto al mecanismo del trauma, las caídas de su propia altura ocuparon el primer lugar (28.5\%), seguidas por trauma deportivo (24.9\%) y caídas de otras alturas (17.2\%). Además, se presentaron accidentes de tránsito, caídas de pasamanos, accidentes en bicicleta, fracturas patológicas y lesiones por agresión. El $84.8 \%$ de los niños se fracturó un solo hueso.

El $52.7 \%(n=1248)$ de las fracturas fueron en el lado izquierdo y solo tres fueron bilaterales $(0.12 \%)$. La mayoría de pacientes tuvieron fracturas cerradas (96.1\%) y solamente un $1.5 \%$ representaron re-fractura.

Tabla 1. Características demográficas y clínicas de los niños con fracturas.

\begin{tabular}{lc}
\hline \multicolumn{1}{c}{ Variable } & $\mathbf{n}=\mathbf{2 4 3 6}$ \\
\hline Edad, mediana (RIC $\left.)^{\mathrm{a}}\right)$ & $10(6-13)$ \\
Género, n (\%) & \\
Masculino & $1597(65.6)$ \\
Femenino & $839(34.4)$ \\
Procedencia, n (\%) & \\
Cali, Valle del Cauca & $1919(78.8)$ \\
Otros municipios del Valle del Cauca & $342(14.0)$ \\
Municipios fuera del Valle del Cauca & $175(7.2)$ \\
Sitio del trauma, n (\%) & \\
Colegio & $503(20.6)$ \\
Escuela de fútbol & $430(17.7)$ \\
Hogar & $389(16.0)$ \\
Vía pública & $368(15.1)$ \\
Parque & $181(7.4)$ \\
Otro centro deportivo & $146(6.0)$ \\
Centro de equitación & $18(0.7)$ \\
Otro & $44(1.8)$ \\
Sin dato & $357(14.7)$ \\
Mecanismo del trauma, n (\%) & \\
Caída de propia altura & \\
Trauma deportivo & $2067(84.8)$ \\
Caída de otras alturas & $369(15.2)$ \\
Accidente de tránsito &
\end{tabular}

Nota: ${ }^{\mathrm{a}}=$ Rango intercuartílico. 
Se hizo tratamiento conservador en $67.1 \%$ de los niños $(\mathrm{n}=1635)$, siendo la reducción cerrada más inmovilización el manejo más frecuente $(\mathrm{n}=902)$, seguida por el manejo con yeso sin necesidad de reducción $(\mathrm{n}=702)$. Por otra parte, de los 752 niños que recibieron tratamiento quirúrgico, la primera forma de manejo fue reducción abierta más clavos $(39.9 \%)$, seguida por reducción cerrada más fijación percutánea (23.7\%) y reducción cerrada más inmovilización bajo anestesia (13.6\%).

Respecto a la distribución por año según género, los niños predominaron durante los cuatro años. En el 2014 fueron el $66.1 \%$, en 2015 el $67.6 \%$, en 2016 el $64.9 \%$ y en 2017 el $63.9 \%$. Además, se observó que para ambos géneros el 2016 fue el año de mayor frecuencia de fracturas (Gráfica 1).

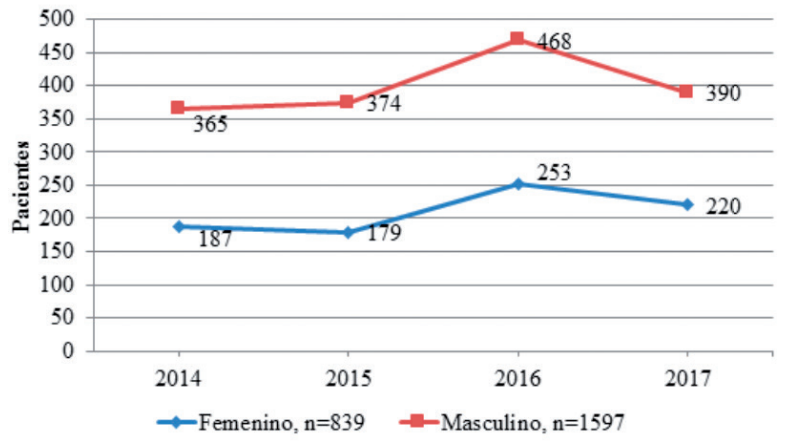

Gráfica 1. Distribución de los niños fracturados por año según el género.

En cuanto a la distribución de las fracturas según el hueso, el radio ocupó el primer lugar. Su frecuencia fue de $32.5 \%$, seguido por el húmero (14.2\%), los huesos de la mano (12.3\%) (carpo, metacarpo y falanges), el cúbito (10.9\%) y la clavícula (7.2\%). La suma de todos los huesos de las extremidades inferiores representó el $21.2 \%$ de las fracturas (fémur, tibia, peroné y pie).

En la distribución por grupo etario, los menores de seis años se fracturaron con mayor frecuencia el húmero, seguido del radio y la clavícula. Los niños entre los 6 y 11 años se fracturaron con mayor frecuencia el radio, seguido del húmero, el cúbito y la mano. Los pacientes entre los 12 y 17 años se fracturaron más el radio, seguido por la mano y el pie. Esto se puede ver en detalle en la Tabla 2 y Gráfica 2.

Los niños entre 6 y 11 años fueron los que más fracturas presentaron, con un total de 991 fracturas (40.7\%) en cuatro años. El segundo lugar lo ocuparon los niños entre los 12-17 años con 887 (36.4\%) y el último lugar fue para los menores de seis años con 558 (22.9\%). Esta distribución se conservó durante los cuatro años del estudio (Gráfica 3).

Tabla 2. Distribución de las fracturas según el hueso y la edad.

\begin{tabular}{lcccc}
\hline \multirow{1}{*}{ Hueso } & \multicolumn{3}{c}{ Edad en años } & Total=2821, \\
\cline { 2 - 5 } & $\begin{array}{r}\mathbf{0 - 5}, \\
\mathbf{n = 6 3 0}\end{array}$ & $\begin{array}{c}\mathbf{6 - 1 1}, \\
\mathbf{n = 1 1 7 3}\end{array}$ & $\begin{array}{c}\mathbf{1 2 - 1 7}, \\
\mathbf{n = 1 0 1 8}\end{array}$ & $\mathbf{n}(\mathbf{\%})$ \\
\hline Radio & 162 & 448 & 307 & $917(32.5)$ \\
Húmero & 171 & 178 & 52 & $401(14.2)$ \\
Mano & 31 & 115 & 201 & $347(12.3)$ \\
Cúbito & 63 & 165 & 79 & $307(10.9)$ \\
Clavícula & 89 & 60 & 54 & $203(7.2)$ \\
Tibia & 42 & 62 & 81 & $185(6.5)$ \\
Pie & 10 & 53 & 96 & $159(5.6)$ \\
Fémur & 42 & 34 & 53 & $129(4.6)$ \\
Peroné & 19 & 45 & 60 & $124(4.4)$ \\
Pelvis-acetábulo & 0 & 6 & 16 & $22(0.8)$ \\
Columna & 1 & 3 & 4 & $8(0.3)$ \\
Otros & 0 & 4 & 15 & $19(0.7)$ \\
\hline
\end{tabular}

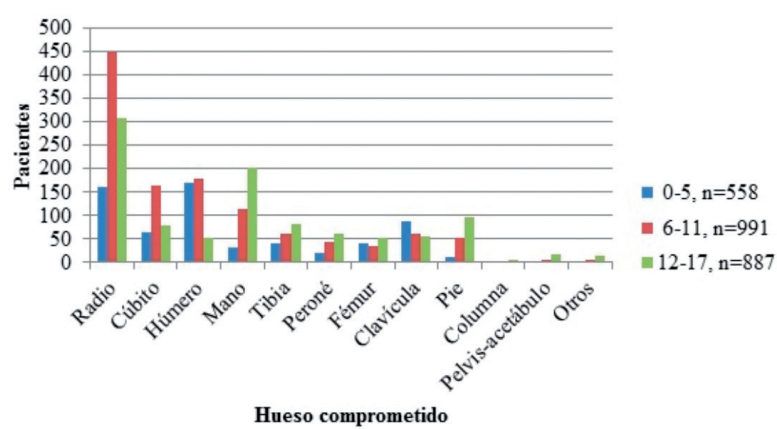

Gráfica 2. Distribución de los niños fracturados según el hueso y la edad.

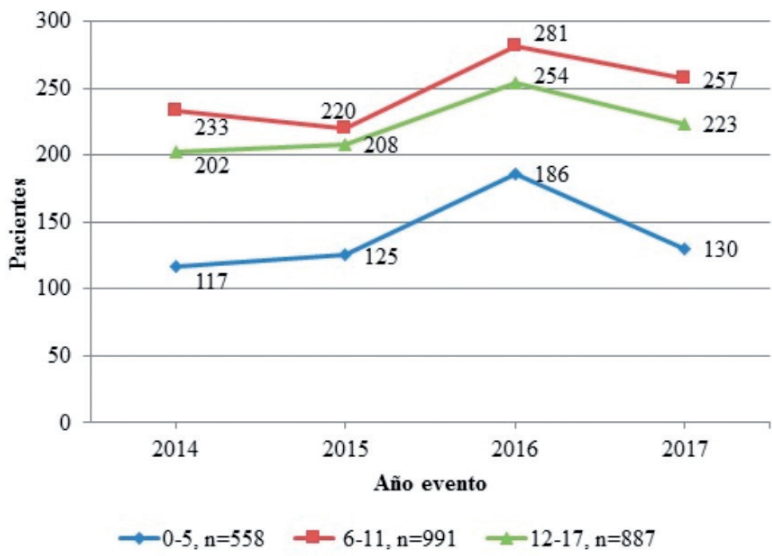

Gráfica 3. Distribución de los niños fracturados por año según la edad.

Durante los cuatro años del estudio consultaron 81,899 niños a la institución por cualquier causa. Esto nos permite hacer un cálculo de incidencia de consulta a la clínica debido a fractura de 29.7/1000 niños por año. 


\section{Discusión}

Este estudio encontró que el género masculino es el más afectado por fracturas en la niñez, similar a lo reportado en la literatura ${ }^{4,16}$. Se ha descrito que el riesgo de presentar una fractura en la vida está entre $42-64 \%$ en niños y $27-40 \%$ en niñas. En diferentes estudios europeos, el riesgo estimado es 1.5 veces mayor en los hombres ${ }^{8,15,16}$. En países en desarrollo se conserva la tendencia, con predominio para el género masculino entre $58-72 \% \%^{1,5,13}$. Esta diferencia entre géneros puede ser atribuida a factores biológicos y sociales, como la tendencia de los niños a realizar actividades físicas de mayor riesgo que las niñas ${ }^{15}$.

La mediana de edad en nuestros pacientes fue de 10 años, comparable con lo publicado por Rennie, et al. y Valerio, et al. quienes reportaron como edades promedio en niños fracturados de 9.7 y 8.8 años, respectivamente ${ }^{6,8}$. Además, se encontró que los pacientes mayores de cinco años presentan una mayor proporción de fracturas, con pico de mayor incidencia entre los 12 y 13 años, hallazgos similares con los encontrados en la literatura ${ }^{6,8}$. Se considera que esto se debe a que los niños mayores de cinco años empiezan a salir del hogar con mayor frecuencia, inician la escolaridad y participan más en actividades deportivas extracurriculares, las cuales aumentan el riesgo de fracturas ${ }^{8,9,15}$.

Al analizar el lugar donde ocurre el trauma, se difiere con la literatura en el orden de frecuencia. En varios estudios reportan el hogar del paciente como el lugar más frecuente donde se fracturan los niños ${ }^{8,11}$. Sin embargo, en el presente estudio fue el colegio, mientras el hogar ocupó el tercer lugar. Este resultado se atribuye a la cantidad de horas del día que los niños permanecen en la jornada escolar, así como al nivel más alto de actividad que ahí se realiza. Por otro lado, puede haber un factor que sesgue este dato y lo aleje de la literatura, ya que en el centro de salud donde se hizo el estudio hay convenio con la mayoría de las polizas escolares y seguros de medicina prepagada, mientras que hay menos convenio con las Empresas Promotoras de Salud (EPS) y las Administradoras de Regimen Subsidiado (ARS). Lo cual podría inclinar la balanza para las consultas por traumas ocurridos en el colegio.

Las caídas son el mecanismo de trauma más frecuente. En la literatura hay reportados porcentajes que van desde $57 \%$ hasta $77 \%$. 6 . En este estudio se encontró que las caídas fueron la causa de fractura de $52.5 \%$ de los niños, siendo la caída desde propia altura la más frecuente.
Respecto al sitio anatómico de la fractura, el miembro superior fue el más comprometido, siendo el radio el hueso más afectado, resultado congruente con lo descrito en otros estudios ${ }^{4,8,15}$. Sin embargo, en este estudio los lactantes y preescolares se fracturaron con mayor frecuencia el húmero, mientras las fracturas de radio son las más frecuentes después de los 6 años y hasta los 17. Por otra parte, las fracturas de los huesos de la mano son las segundas en frecuencia después de los 11 años, similar a lo reportado en otras investigaciones ${ }^{6,8}$.

Referente a la lateralidad, se ha descrito que las fracturas en niños ocurren generalmente en el lado izquierdo (59-68\%), con una relación izquierda:derecha de 1.3:126-28. Esta distribución se puede explicar por la mayor frecuencia de dominancia diestra. Durante la actividad física es el lado dominante el que realiza la acción, mientras la extremidad opuesta es usada para protección durante el trauma ${ }^{29}$. Además, se ha descrito una mayor fragilidad ósea o una inmadurez en la coordinación neuromuscular que hace al lado izquierdo menos resistente a manejar lesiones traumáticas ${ }^{27}$. Este estudio obtuvo resultados acordes a la literatura, siendo el lado izquierdo el más comprometido (52\%). Por otro lado, se identificó que la mayoría de los pacientes $(96.1 \%)$ presentaron fracturas cerradas, similar a la literatura que atribuye a las fracturas abiertas menos del $10 \%$ de los $\operatorname{casos}^{20,24,25}$.

Las fracturas en niños generalmente tienen una evolución favorable debido al alto potencial de remodelación en edades tempranas, es por esto que la mayoría de las fracturas pediátricas se manejan de forma conservadora (sin cirugía). El manejo quirúrgico se indica solamente en el $10-25 \%$ de todos los $\operatorname{casos}^{20,24,25,30,31}$. Este estudio concuerda con la tendencia mundial de manejar estas fracturas de forma no quirúrgica, sin embargo, la proporción de tratamiento quirúrgico $(32.9 \%)$ es mayor que la reportada en la literatura previamente mencionada. Esto puede estar relacionado con que la institución donde se realizó incluye la atención de pacientes de alta complejidad.

La incidencia observada es similar a la literatura, que está alrededor de 20 niños fracturados por cada 1000 niños. Además, es superior a lo reportado en los años 90 en la ciudad de Cali $^{6-8,10}$.

A partir de los resultados surgen preguntas. ¿Qué estrategias se pueden implementar para disminuir el riesgo de fracturas en niños? debido a que el $52.5 \%$ de las fracturas fueron por caídas, un programa enfocado en la educación de padres, profesores y cuidadores sobre mecanismos y sitios donde es más frecuente que 
pueda ocurrir un trauma, podría ayudar a disminuir la incidencia. También se podrían realizar campañas informativas sobre el uso de elementos de protección personal cuando se realicen actividades deportivas, incrementar la supervisión de adultos responsables durante el tiempo de juego en casa y en el colegio. Finalmente, desde el gobierno se debe reforzar la educación sobre seguridad vial para prevenir las fracturas por accidentes de tránsito ${ }^{6}$.

Como limitantes adicionales de este estudio, debido al tipo de diseño escogido no se pueden establecer factores de riesgo para las fracturas, solamente asociaciones. La ausencia de seguimiento no permite evaluar los desenlaces clínicos. Como se mencionaba previamente, puede haber un sesgo de selección por el tipo de aseguradoras que se atienden en la institución y por el alto nivel de complejidad de la misma. También puede haber sesgo de información, al tratarse de una revisión retrospectiva de historias clínicas.

\section{Conclusiones}

Las fracturas en niños siguen siendo un motivo frecuente de consulta en ortopedia y traumatología, con una incidencia diaria de 1.7 consultas por fractura en un centro de alto nivel de complejidad y una incidencia anual de 29.7 fracturas por cada 1000 niños. El género masculino es el más afectado y el radio es el principal hueso comprometido. La mayoría de estas fracturas son manejadas de forma conservadora. Por último, las refracturas ocupan un porcentaje muy bajo, similar a la literatura ${ }^{32}$.

\section{Agradecimientos}

Agradecemos al Centro de Investigaciones Clínicas (CIC) de la Fundación Valle del Lili por el apoyo metodológico brindado durante la realización de este manuscrito. Además, queremos agradecer a Andrés Mauricio Castro, estadístico del CIC, por su colaboración con el análisis de datos.

\section{Consideraciones éticas}

Esta investigación se realizó de forma retrospectiva y sin ningún tipo de intervención en los sujetos estudiados, limitándose exclusivamente a la revisión de historias clínicas y las imágenes diagnósticas, por lo que según la legislación colombiana (Resolución 8430 de 1993) es considerada una investigación sin riesgo. Adicionalmente, esta investigación fue aprobada por el Comité de Ética e Investigación Biomédica de nuestra institución, por medio del acta 16 del 11 de agosto de 2017, número del protocolo 1174.

\section{Conflicto de intereses}

Los autores declaran no tener conflicto de intereses. Este estudio no recibió financiación.

\section{Referencias}

1. Fiorentino JA, Molise C, Stach P, Cendero P, Solla MM, Hoffman E, et al. Trauma en pediatría. Estudio epidemiológico en pacientes internados en el Hospital de Niños "Ricardo Gutiérrez". Arch Argent Pediatr. 2015; 113(1): 12-20. doi: https://doi. org/10.5546/aap.2015.12.

2. Neira JA, Bosque L, Zengotita S. Informe estadístico sobre trauma. Buenos Aires: Gobierno de la ciudad de Buenos Aires, Sociedad Argentina de medicina y cirugía del trauma. 2000.

3. Iñon AE, Rocca Rivarola M, Herscovici C, Alessandria JC. Morbilidad de los accidentes en pacientes pediátricos y sus familias. Arch Argent Pediatr. 1993; 91(4): 202-210.

4. Jeddi M, Dabbaghmanesh $\mathrm{MH}$, Kharmandar A, Ranjbar Omrani G, Bakhshayeshkaram M. Prevalence of fracture in healthy Iranian children aged 9-18 years and associated risk factors; a population based study. Bull Emerg Trauma. 2017; 5(1): 29-35.

5. Franco-Abreu G, Rodríguez CP. Los accidentes en los niños. Un estudio epidemiológico. Rev Mex Pediatr. 2000; 67(1): 9-11.

6. Rennie L, Court-Brown CM, Mok JY, Beattie TF. The epidemiology of fractures in children. Injury. 2007; 38(8): 913-922. doi: https://doi.org/10.1016/j. injury.2007.01.036.

7. Thandrayen K, Norris SA, Micklesfield LK, Pettifor JM. Fracture patterns and bone mass in South African adolescent- Mother pairs: The birth to twenty cohort. Osteoporos Int. 2014;25(2):693-700. doi: https://doi.org/10.1007/s00198-013-2477-4.

8. Valerio G, Gallè F, Mancusi C, Di Onofrio V, Colaprieto M, Guida P, et al. Pattern of fractures across pediatric age groups: Analysis of individual and lifestyle factors. BMC Public Health. 2010; 10: 656. doi: https://doi.org/10.1186/1471-2458-10656.

9. Christoffersen T, Ahmed LA, Winther A, Nilsen OA, Furberg AS, Grimnes G, et al. Fracture incidence rates in Norwegian children, The Tromsø Study, Fit Futures. Arch Osteoporos. 2016; 11(1): 40. doi: https://doi.org/10.1007/s11657-016-0294-z. 
10. Danseco ER, Miller TR, Spicer RS. Incidence and costs of 1987-1994 childhood injuries: demographic breakdowns. Pediatrics. 2000; 105(2): E27. doi: https://doi.org/10.1542/peds.105.2.e27.

11. Alomran AK, Bubshait DA, Sadat-Ali M. Epidemiology of pediatric fractures and dislocations: Analysis of in-patients. Bahrain Med Bull. 2012; 34(4).

12. Wegmann H, Orendi I, Singer G, Eberl R, Castellani $\mathrm{C}$, Schalamon J, et al. The epidemiology of fractures in infants - Which accidents are preventable? Injury. 2016; 47(1): 188-191. doi: https://doi.org/10.1016/j. injury.2015.08.037.

13. Saw A, Fadzilah N, Nawar M, Chua YP. Pattern of Childhood Fractures in a developing country. Malaysian Orthop J. 2011; 5(1): 13-16. doi: https:// doi.org/10.5704/MOJ.1103.003.

14. De Pablos J, González P. Fracturas Infantiles: conceptos y principios. 2da edición. España: Global HELP, 2010: p.34.

15. Hedström EM, Svensson O, Bergström U, Michno P. Epidemiology of fractures in children and adolescents: Increased incidence over the past decade: A population-based study from northern Sweden. Acta Orthop. 2010; 81(1): 148-153. doi: https://doi.org/10.3109/17453671003628780.

16. Mora FG, Gustavo F, Carriedo JM, Bustamante BB, Mejía LC, López A, et al. Fracturas más frecuentes en niños en el Hospital Regional General Ignacio Zaragoza del ISSSTE. Esp Méd Quir. 2012; 17(3): 175-178.

17. Deakin DE, Crosby J, Moran CG, Chell J. Childhood fractures requiring inpatient management. Injury. 2007; 38(11): 1241-1246. doi: https://doi. org/10.1016/j.injury.2007.05.023.

18. Mäyränpää M. Fractures in Children : epidemiology and associated bone health characteristics. Helsinki: University of Helsinki. 2012.

19. Mansoor K, Shahnawaz S, Ahmad A, Arif MM, Hamza M. Epidemiology of childhood fractures in the city of Karachi. J Ayub Med Coll Abbottabad. 2015; 27(3): 608-612.

20. Concha J, Gerstner J, Gallón L, Bárcenas, C. Patrón de fracturas en niños en el Hospital Universitario de Cali. Rev Col Or Tra. 1990; 4(3): 247-251.

21. Goulding A. Risk Factors for Fractures in normally active children and adolescents. Med Sport Sci. 2007; 51: 102-120. doi: https://doi. org/10.1159/000103007.

22. Yeh FJ, Grant AM, Williams SM, Goulding A. Children who experience their first fracture at a young age have high rates of fracture. 2006; 17(2): 267-272. doi: https://doi.org/10.1007/s00198-005- 2009-y.

23. Helenius I, Lamberg TS, Kääriäinen S, Impinen A, Pakarinen MP. Operative treatment of fractures in children is increasing. A population-based study from Finland. J Bone Joint Surg Am. 2009; 91(11): 2612-2616. doi: https://doi.org/10.2106/ JBJS.H.01519.

24. Vega R, Piñeros DF, Amador JS. Descripción epidemiológica y evaluación de los desenlaces de interés de las fracturas del tercio superior del húmero en niños. Rev Fac Med. 2014; 62(3): 347-353. doi: https://doi.org/10.15446/revfacmed.v62n3.39486.

25. Riascos GA. Descripción epidemiológica de las fracturas de tibia y peroné en el Hospital de la Misericordia en los últimos 5 años. Tesis de grado: Universidad Nacional de Colombia. 2012.

26. Landin LA. Fracture patterns in children. Analysis of 8,682 fractures with special reference to incidence, etiology and secular changes in a Swedish urban population 1950-1979. Acta Orthop Scand Suppl. 1983; 202: 1-109. doi: https://doi. org/10.3109/17453678309155630.

27. Mortensson W, Thönell S. Left-side dominance of upper extremity fracture in children. Acta Orthop Scand. 1991; 62(2): 154-155. doi: https://doi. org/10.3109/17453679108999245. Artículo de revista

28. Hassan FO. Hand dominance and gender in forearm fractures in children. Strategies Trauma Limb Reconstr. 2008; 3(3): 101-103. doi: https://doi. org/10.1007/s11751-008-0048-6.

29. Rohl L. On fractures through the radial condyle of the humerus in children. Acta Chir Scand. 1952; 104(1): 74-80.

30. Salunkhe RM, Deshmukh A, Kumar Sumeet, Kandari A. Conservative vs . surgical intervention in diaphyseal forearm fractures in age group 1 to 15 years : A prospective study. Int. j. orthop. sci. 2018; 4(3): 251-254. doi: https://doi.org/10.22271/ ortho.2018.v4.i3e.43.

31. Kinney MC, Nagle D, Bastrom T, Linn MS, Schwartz AK, Pennock AT. Operative versus conservative management of displaced tibial shaft fracture in adolescents. J Pediatr Orthop. 2016; 36(7): 661-666. doi: https://doi.org/10.1097/ BPO.0000000000000532.

32. Tisosky AJ, Werger MM, McPartland TG, Bowe JA. The Factors Influencing the refracture of pediatric forearms. J Pediatr Orthop. 2015; 35(7): 677-681. doi: https://doi.org/10.1097/BPO.0000000000000355. 